\title{
Anabases
}

ANABASES Traditions et réceptions de l'Antiquité

$20 \mid 2014$

Varia

\section{Les archives scientifiques de Paul Perdrizet à l'université de Lorraine (Nancy)}

Samuel Provost

\section{(2) OpenEdition}

1 Journals

Édition électronique

URL : http://journals.openedition.org/anabases/5116

DOI : 10.4000/anabases. 5116

ISSN : 2256-9421

Éditeur

E.R.A.S.M.E.

Édition imprimée

Date de publication : 1 novembre 2014

Pagination : 385-400

ISSN : 1774-4296

\section{Référence électronique}

Samuel Provost, «Les archives scientifiques de Paul Perdrizet à l'université de Lorraine (Nancy)»,

Anabases [En ligne], 20 | 2014, mis en ligne le 01 novembre 2017, consulté le 20 octobre 2019. URL

http://journals.openedition.org/anabases/5116 ; DOI : 10.4000/anabases.5116

Ce document a été généré automatiquement le 20 octobre 2019.

(c) Anabases 


\title{
Les archives scientifiques de Paul Perdrizet à l'université de Lorraine (Nancy)
}

\author{
Samuel Provost
}

1 Le département d'archéologie et d'histoire de l'art de l'université de Lorraine (Nancy) conserve un fonds d'archives scientifiques, pédagogiques et administratives important se rapportant à l'Institut d'archéologie classique et à son créateur, le professeur Paul Perdrizet (1870-1938). Une partie correspond aux archives propres des différentes incarnations administratives de la section d'archéologie et d'histoire de l'art de la faculté des Lettres, depuis sa création en 1901. S'y est ajoutée en 1981 la donation par les héritiers de la veuve de Paul Perdrizet, Lucile Perdrizet-Gallé, d'un lot important d'archives scientifiques et personnelles. Depuis cette date, ce fonds était resté totalement inexploité; il n'avait pas été inventorié alors qu'il représente une masse documentaire non négligeable : 29 cartons d'archives manuscrites et une dizaine de dossiers supplémentaires, 1400 tirés à part, plus de 120 boîtes de plaques de verre photographiques, pour un total supérieur à 9000 clichés, 2400 tirages photographiques montés sur carton. Depuis la fin de 2012, un programme de valorisation du fonds a commencé, avec pour objectif l'inventaire, le reconditionnement, la numérisation et la mise en ligne rapide d'une partie des documents, dont la correspondance scientifique. Bien que la documentation iconographique reste entièrement à étudier, l'achèvement du classement des archives manuscrites permet de présenter un premier bilan sur l'intérêt de ces archives.

2 L'originalité du fonds tient à la personnalité même de Paul Perdrizet ${ }^{1}$, aux multiples facettes : normalien, agrégé de Lettres et ancien membre de l'École française d'Athènes, cet universitaire allie une profonde érudition, qui impressionne même ses pairs, une vive curiosité intellectuelle et une énergie hors du commun. Archéologue et épigraphiste, philologue, historien de l'art et médiéviste, il ne s'est pas cantonné à un domaine de recherche ni à une seule période. Ses travaux ont porté aussi bien sur la sculpture grecque archaïque, la religion thrace, la numismatique macédonienne, 
l'épigraphie grecque classique, que l'iconographie chrétienne du Moyen Âge occidental, l'architecture byzantine, le calendrier liturgique catholique médiéval ou la peinture de la Renaissance italienne, pour ne citer qu'une partie de ses thèmes de recherche. Son œuvre scientifique compte plus de deux cent soixante-dix titres répartis sur quarante et un ans d'activité, malgré l'hiatus de la Première Guerre mondiale ${ }^{2}$.

La vie universitaire n'est toutefois qu'un versant de l'activité de Paul Perdrizet. Dès son arrivée à Nancy en 1899, il s'investit dans la vie associative et politique locale, devenant le président de l'Union de la jeunesse lorraine, et il joue un rôle clef dans le développement de l'université populaire de Nancy. C'est grâce à cet engagement qu'il fait la connaissance d'émile Gallé, le maître verrier et ébéniste, fondateur de l'École de Nancy et théoricien de l'Art Nouveau en France. En 1906, moins de deux ans après la mort précoce de ce dernier, il en épouse l'une des filles, Lucile. Progressivement, il apporte son aide à sa veuve, Henriette Grimm-Gallé, pour sauvegarder l'héritage du maître. Il entre en 1906 au comité directeur de l'École de Nancy, désormais dirigée par le peintre Victor Prouvé. Après la Première Guerre mondiale, bien que nommé professeur d'archéologie à l'université de Strasbourg, sur l'ancienne chaire d'Adolf Michaelis qu'en Alsacien revanchard il avait ardemment désirée, il continue de résider à Nancy : il assume en effet la direction effective des Établissements Gallé jusqu'à leur fermeture définitive en 1936.

\section{Les archives manuscrites}

4 Les archives manuscrites conservées à l'université de Nancy ne représentent à l'évidence qu'une fraction de l'activité scientifique de Perdrizet. Le laps de temps important qui s'est écoulé, d'une part entre sa mort et celle de sa veuve (respectivement en 1938 et 1981), d'autre part depuis le transfert des archives dans les locaux de l'université à cette occasion, a rendu en effet possibles des prélèvements antérieurs. Ce délai explique également que les informations à ce sujet soient rares, car les principaux acteurs de la donation sont maintenant décédés: Françoise-Thérèse Charpentier, spécialiste de l'Art nouveau, qui connaissait Lucile Perdrizet et qui avait perçu l'intérêt de cet héritage ; Jean Bourgogne ${ }^{3}$, l'héritier de Lucile Gallé qui avait consenti le legs informel des archives scientifiques de Paul Perdrizet. On sait néanmoins par Lucile Perdrizet que des lettres des archives familiales Gallé furent détruites, et le même sort a dû être réservé également à une partie de la correspondance de Paul Perdrizet ${ }^{4}$.

5 Faute d'informations de première main, l'histoire du fonds doit être reconstituée d'après quelques éléments intrinsèques (étiquettes autographes, répartition des dossiers entre les cartons) ainsi que par les archives familiales. Le classement originel des cartons serait intervenu vers 1930-1932. Il consista en un archivage sommaire des dossiers de recherche réunis depuis la fin des années 1890 ainsi que dans le rangement thématique des tirés à part reçus, dans une seconde série de cartons identiques à ceux utilisés pour les manuscrits. Il fut complété progressivement jusqu'en 1938 par les dossiers des nouveaux travaux, publiés ou non. Après la disparition de son mari, Lucile Perdrizet intervint à plusieurs reprises pour reclasser des manuscrits et imprimés : elle ajouta notamment au fonds trois cartons en 1956 après que la vente viagère (ou assimilée) de la maison familiale l'eut contrainte à de nouveaux rangements. 
6 Chaque carton d'archives manuscrites comprend ainsi, en général, des liasses de notes préparatoires, des brouillons d'articles, rarement le manuscrit final, la documentation photographique (plaques de verre et tirages papier), la correspondance afférente et les tirés à part des principales études utilisées - parfois celui de l'article publié, avec des annotations de correction ou des addenda.

7 La comparaison entre le contenu des dossiers conservés, d'après l'inventaire préliminaire réalisé, et la bibliographie du savant conduit aux remarques suivantes : la deuxième partie de sa carrière, après la Première Guerre mondiale, est beaucoup mieux représentée dans le fonds, comme il est attendu : les documents les plus récents sont les plus nombreux, aussi bien pour les archives de travail que pour les documents épistolaires. Les pièces les plus anciennes du fonds datent de 1895, de son premier séjour à l'École française d'Athènes. Le fonds couvre donc l'ensemble de sa carrière, mais de façon inégale. Les archives manuscrites antérieures à la Première Guerre mondiale sont moins nombreuses et consistent essentiellement en dossiers de notes classés par thèmes ou par publication. Ce sont à la fois les dossiers de préparation de ses articles et les notes de terrain.

8 Toutes les archives de ses missions archéologiques ne sont pas présentes: pour la Macédoine, le fonds comprend les archives intégrales de la grande mission de l'été 1899 (carnets de terrain, lettres et rapports de mission envoyés au directeur de l'École française d'Athènes, Théophile Homolle) $)^{5}$, mais celles de 1894 et 1901 manquent à l'appel. Les autres missions qu'il a conduites lors de son séjour à l'École française d'Athènes, à savoir dans l'ordre chronologique, en Asie mineure (1895), à Chypre (1896), en Syrie (1896 et 1902), en Locride (1897) et en Angleterre (1897-1898) manquent ou n'ont pas encore été localisées. Les archives de l'École française d'Athènes permettent de combler certaines de ces lacunes, essentiellement pour sa participation à la « Grande fouille » de Delphes, où il fut l'un des principaux collaborateurs de Théophile Homolle. Le site de Delphes n'est en effet représenté dans les archives nancéiennes que par une documentation photographique. En revanche, les deux missions postérieures à la Première Guerre mondiale menées en Syrie (1924-1925), avec Henri Seyrig, sont l'objet de deux cartons avec l'essentiel des archives de travail (carnets de terrain, cartes topographiques, estampages, brouillons du manuscrit inédit). Enfin, les travaux en Égypte sont inégalement représentés : cette fois, ce sont les missions les plus anciennes (1909 et 1912 pour les inscriptions d'Abydos et les collections antiques de Daniel Fouquet) qui sont les mieux conservées, même si une partie de la documentation se trouve à Paris dans les archives de l'ancienne Bibliothèque Jacques Doucet, puisque c'est ce dernier qui en finança la publication. Les archives des missions égyptiennes de la deuxième partie de sa carrière, concernant les terres cuites du musée du Caire (1928), et surtout les fouilles de la nécropole d'Hermopolis Magna avec l'université du Caire $(1933,1934,1936)$ sont logiquement manquantes : elles furent confiées à Pierre Jouguet dès juillet 1938 pour servir à la publication posthume des études correspondantes ${ }^{6}$.

Le fonds comprend par ailleurs les dossiers de préparation de certaines monographies publiées par Paul Perdrizet : ses deux thèses, La Vierge de Miséricorde (1908) et Étude sur le Speculum Humanae Salvationis (1908); Les graffites grecs du Memnonion d'Abydos (1919) (avec les estampages); le Negotium perambulans in tenebris (1922); Le calendrier parisien à la fin du Moyen Âge (1933).

10 Enfin, plusieurs dossiers contiennent les archives relatives à la série des grands colloques internationaux auxquels a participé Paul Perdrizet: les colloques d'études 
byzantines de Bucarest (1924), Belgrade (1927) et Sofia (1934), le congrès international d'archéologie d'Alger (1930), le congrès d'archéologie chrétienne de Ravenne (1932). Ces dossiers sont d'autant plus précieux que Perdrizet confiait rarement le texte de ses communications aux volumes des actes des congrès et préférait les publier séparément. Or, certaines communications sont restées inédites et ne sont conservées que dans le fonds de l'université de Lorraine.

11 La correspondance professionnelle présente dans le fonds connaît la même distribution que les manuscrits de travail : à l'exception de deux cartons plus spécifiques et datant de la fin des années 1930, elle est en effet dispersée dans les cartons d'archives. Abondante pour les dernières années, elle est plus rare pour les années 1900-1920 et presque entièrement manquante pour les années antérieures, à l'exception de quelques dossiers précis. L'inventaire provisoire qui en a été réalisé permet de comptabiliser 1245 lettres qui proviennent de 322 correspondants, avec une distribution très inégale. Les 15 correspondants les mieux représentés totalisent 352 lettres soit $34 \%$ du total, tandis que 170 ne sont connus que par une seule lettre. Même pour les correspondances les plus développées, la partie conservée n'est qu'une fraction de l'ensemble des courriers échangés : c'est ce que démontre la comparaison avec les fonds extérieurs possédant la correspondance active de Paul Perdrizet. L'exemple extrême est ainsi celui de la correspondance avec le critique d'art et bibliothécaire René Jean, conservée à l' $\mathrm{INHA}^{7}$ : elle compte 467 lettres écrites par Paul Perdrizet entre 1905 et 1938, alors que les différentes archives de ce savant ne réunissent que 13 lettres de René Jean ${ }^{8}$. D’autres correspondances sont heureusement mieux conservées tout en restant lacunaires, avec René Dussaud, Pierre Jouguet ou encore Henri Seyrig. La numérisation de cet ensemble est achevée et la préparation de la base de données qui en permettra la publication est en cours.

12 L'équivalent de deux cartons d'archives constitue un fonds d'origine différente, les archives administratives de l'Institut d'archéologie classique, fondé par Paul Perdrizet en 1901-1902. La conservation de ces pièces est à mettre au compte de son successeur à la chaire d'archéologie et d'histoire de l'art, Marcel Bulard, qui les a classées avec celles de sa propre activité administrative. Elles n'ont donc jamais quitté l'université et elles ont suivi, dans des circonstances encore non élucidées, le transfert de la faculté des lettres depuis le palais universitaire, place Carnot, jusqu'à l'actuel campus, vers 1963. Ces archives comportent notamment un nombre important de pièces relatives à la constitution de l'instrumentum d'histoire de l'art et d'archéologie : les commandes de moulages pour la gypsothèque universitaire, de reproductions photographiques, de livres pour la bibliothèque de l'Institut, les demandes de dépôts d'œuvres pour le musée archéologique.

13 Ce petit fonds institutionnel est d'autant plus important que l'enseignement est par ailleurs mal représenté dans l'ensemble des archives professionnelles de Paul Perdrizet : les cours et conférences donnés aux universités de Nancy et de Strasbourg manquent presque totalement, à l'exception de quelques notes préparatoires, éparpillées dans divers dossiers. Seuls les enseignements de langue et de littérature grecques, correspondant pour l'essentiel à la période antérieure à 1909, sont en partie conservés, sous la forme d'épais cahiers. L'hypothèse la plus probable est que pour ses cours d'histoire de l'art et d'archéologie, il ne s'appuyait que sur des notes sommaires qu'il n'a pas conservées. 


\section{Les imprimés}

d

directement reliée aux archives manuscrites du fonds qui en constituent une partie des notes préparatoires et brouillons. Or le fonds nancéien ne contient qu'une fraction de ces publications: si certains articles sont encore conservés à plusieurs dizaines d'exemplaires, un grand nombre manque complètement à l'appel. Cette absence s'explique par deux raisons : durant sa carrière, Paul Perdrizet a largement distribué ses tirés à part, comme l'attestent ses archives comme sa correspondance, et il a naturellement privilégié l'envoi des travaux qu'il jugeait les plus importants. Certains éditeurs ne remettant qu'un nombre limité d'exemplaires des articles ou monographies à leurs auteurs, Paul Perdrizet a rapidement épuisé ses exemplaires personnels : en 1911, par exemple, il signale à René Jean qu'il n'a reçu qu'un exemplaire de la Bibliographie lorraine, pourtant parue dans la revue de l'université de Nancy, les Annales de l'Est, et qu'il va devoir en acheter un exemplaire pour le remettre à la bibliothèque d'art et d'archéologie de J. Doucet.

Une deuxième explication de ces lacunes est la dispersion de la bibliothèque personnelle de Paul Perdrizet par sa veuve, pour des raisons financières, entre 1949 et 1951. L'histoire complexe de cette vente peut être reconstituée d'après les archives familiales : le catalogue manuscrit de la bibliothèque de Paul Perdrizet montre qu'elle ne contenait déjà plus qu'un exemplaire, au maximum, de ses propres publications. Les ouvrages conservés aujourd'hui dans le fonds nancéien sont donc apparemment ceux qui ne trouvèrent pas preneur dans ces ventes ou bien qui échappèrent par hasard à l'envoi au dépôt parisien où elles eurent lieu. Il faut encore y ajouter les publications, dons directs du savant ou de ses héritiers, présentes dans les différentes bibliothèques universitaires nancéiennes, en particulier celle de l'UfR Histoire-Géographie, ultime descendante de la bibliothèque de l'Institut d'archéologie classique créée par Perdrizet, et la bibliothèque du centre de recherches Édouard Will.

Quant à la deuxième catégorie des imprimés, les articles, et parfois les monographies, envoyés en hommage à Paul Perdrizet par ses correspondants scientifiques, ils se répartissent entre 20 boîtes cartonnées, pour un total de 1400 titres selon un classement thématique et géographique très général, dont Paul Perdrizet est lui-même l'auteur. Un deuxième lot de brochures, au nombre de 400, est dispersé dans les cartons d'archives scientifiques, où elles sont classées avec les archives manuscrites des recherches correspondantes.

Ce premier inventaire des archives Perdrizet appelle des recherches complémentaires pour tenter de localiser certains dossiers qui en furent retirés après sa mort ou les correspondances actives complétant les séries épistolaires conservées. Pour lacunaire qu'il soit, le fonds n'en comporte pas moins en effet des documents importants et surtout des travaux inédits qui permettront de réévaluer l'œuvre de ce savant. 
Paul Perdrizet à Bâle en août 1908 (archives familiales)

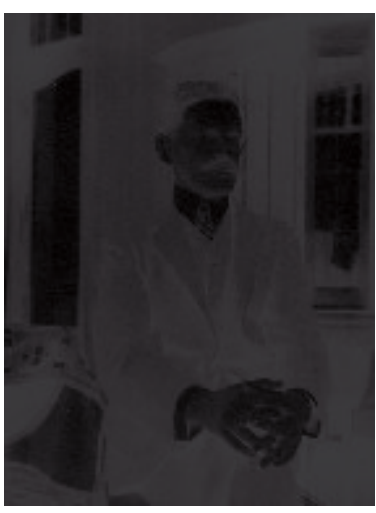

\section{NOTES}

1. L'étude biographique la plus complète de P. Perdrizet reste encore l'éloge funèbre prononcé par Ch. Picard à l'Académie des inscriptions et belles-lettres peu de jours après sa mort en 1938 (CH. PICARD, «ÉLOGE FUNÈBRE DE M. PAUL PERDRIZET, MEMBRE DE L'ACADÉMIE », COMPTES RENDUS DE L'ACADÉMIE DES INSCRIPTIONS ET BELLES LETTRES, 1938, VOL. 82, №3, P. 270-280). LA GRANDE MAJORITÉ DES RARES NOTICES BIOGRAPHIQUES CONSACRÉES À CE SAVANT EN DÉRIVENT ÉTROITEMENT.

2. Là encore, c'est l'esquisse bibliographique donnée par $\mathrm{Ch}$. Picard qui reste la référence : $\mathrm{CH}$. PICARD, « PAUL PERDRIZET (1870-1938) », REVUE ARCHÉOLOGIQUE, 1938, VOL. 12, P. 236-237.

3. Sa fille, Mme Jacqueline Amphoux, donc la petite-nièce de Paul et Lucile Perdrizet, reste un témoin important de cette histoire. Je la remercie vivement pour l'aide inestimable qu'elle m'apporte dans l'étude des archives Perdrizet. Mes remerciements vont également à Francis Croissant et Yves Grandjean, qui se sont succédé sur la chaire d'archéologie grecque de l'université de Nancy, pour les renseignements qu'ils m'ont donnés sur le transfert des archives en 1981.

4. L'information a été recueillie par F. Th. Charpentier et reprise dans É. GALLÉ ET R. MARX, LETTRES POUR L'ART, CORRESPONDANCE 1882-1904, ÉD. F.-TH. CHARPENTIER, G. BARBIER-LUDWIG ET B. PONTON, STRASBOURG, 2006, N. 8 P. 38.

5. Paul Perdrizet a probablement repris ces documents en 1900 ou 1901 lorsqu'il est rappelé pour un an à Athènes.

6. Si les fouilles d'Hermoupolis furent bien publiées (S. GABRA, RAPPORT SUR LES FOUILLES D'HERMOUPOLIS OUEST (TOUNA EL-GEBEL), INSTITUT FRANÇAIS D'ARCHÉOLOGIE ORIENTALE DU CAIRE, 1941), LE MANUSCRIT DU CATALOGUE DES TERRES CUITES DU MUSÉE DU CAIRE, CONFIÉ À ÉTIENNE DRIOTON, RESTA INÉDIT.

7. Autographes René Jean, 144-145, nº 520 à 986, INHA.

8. Dix sont conservées dans les archives de l'UL ET TROIS DANS LES ARCHIVES FAMILIALES À PARIS. 


\section{AUTEUR}

\section{SAMUEL PROVOST}

Maître de conférences d'archéologie et d'histoire de l'art

Pôle archéologique universitaire (EA 1132 HISCANT-MA),

MSH Lorraine

Université de Lorraine

samuel.provost@univ-lorraine.fr 